\title{
A POSITIVITY CONJECTURE FOR JACK POLYNOMIALS
}

\author{
Michel LASSALLE
}

ABSTRACT. We present a positivity conjecture for the coefficients of the development of Jack polynomials in terms of power sums. This extends Stanley's ex-conjecture about normalized characters of the symmetric group. We prove this conjecture for partitions having a rectangular shape.

\section{Introduction}

A finite weakly decreasing sequence of positive integers $\lambda=\left(\lambda_{1}, \ldots, \lambda_{r}\right)$ is called a partition with length $l(\lambda)=r$ and weight $|\lambda|=\sum_{i} \lambda_{i}$. Let $n$ be a fixed positive integer and $\mathfrak{S}_{n}$ the group of permutations of $n$ letters. The irreducible representations of $\mathfrak{S}_{n}$ are labelled by partitions with weight $n$. Let $\chi^{\lambda}$ denote the corresponding character.

By identification of the cycle decomposition of a permutation with a partition, any partition $\rho$ with weight $n$ defines a conjugacy class of $\mathfrak{S}_{n}$. Let $\mu$ be a partition with weight $k \leq n$, and $\left(\mu, 1^{n-k}\right)$ the partition obtained by adding $n-k$ parts 1 . The normalized character $\hat{\chi}_{\mu, 1^{n-k}}^{\lambda}$ is defined by

$$
\widehat{\chi}_{\mu, 1^{n-k}}^{\lambda}=\frac{n !}{(n-k) !} \frac{\chi_{\mu, 1^{n-k}}^{\lambda}}{\chi_{1^{n}}^{\lambda}},
$$

where $\chi_{\rho}^{\lambda}$ is the value of $\chi^{\lambda}$ on the conjugacy classs $\rho$, and $\chi_{1^{n}}^{\lambda}=\operatorname{dim} \lambda$ is the dimension of the irreducible representation $\lambda$.

Let $\boldsymbol{p}=\left(p_{1}, \ldots, p_{m}\right)$ and $\boldsymbol{q}=\left(q_{1}, \ldots, q_{m}\right)$, with $q_{1} \geq \cdots \geq q_{m}$, be two sequences of $m$ positive integers. We denote $-\boldsymbol{q}=\left(-q_{1}, \ldots,-q_{m}\right)$ and $\boldsymbol{p} \times \boldsymbol{q}$ the partition which is the union of $m$ rectangles of sizes $p_{i} \times q_{i}$, namely

$$
\boldsymbol{p} \times \boldsymbol{q}=(\underbrace{q_{1}, \ldots, q_{1}}_{p_{1} \text { times }}, \underbrace{q_{2}, \ldots, q_{2}}_{p_{2} \text { times }}, \ldots) .
$$

The following result was conjectured by Stanley [17] and proved by Féray [1], under a more precise statement which was also conjectured by Stanley [18].

Theorem. For $\lambda=\boldsymbol{p} \times \boldsymbol{q}$ the normalized character $(-1)^{k} \widehat{\chi}_{\mu, 1^{n-k}}^{\lambda}$ is a polynomial in the indeterminates $(\boldsymbol{p},-\boldsymbol{q})$ with nonnegative integer coefficients.

The purpose of this paper is to present a conjectured extension of this property, in the framework of Jack polynomials.

The family of Jack polynomials $J_{\lambda}(\alpha)$ is indexed by partitions. It forms a basis of the algebra of symmetric functions with rational coefficients in some positive real

Received by the editors April 10, 2007. 
parameter $\alpha$. We consider the transition matrix between this basis and the classical basis of power sums $p_{\rho}$. Namely we write

$$
J_{\lambda}(\alpha)=\sum_{|\rho|=|\lambda|} \theta_{\rho}^{\lambda}(\alpha) p_{\rho} .
$$

Let $\mu$ be a partition of weight $k=|\mu| \leq|\lambda|=n$. Using multiplicities, we denote $\mu=\left(1^{m_{1}(\mu)}, 2^{m_{2}(\mu)}, \ldots\right)$ and $z_{\mu}=\prod_{i \geq 1} i^{m_{i}(\mu)} m_{i}(\mu)$ !. We consider the quantity

$$
\vartheta_{\mu}^{\lambda}(\alpha)=z_{\mu} \theta_{\mu, 1^{n-k}}^{\lambda}(\alpha)
$$

for which we conjecture the following positivity property.

Conjecture 1. Let $\lambda=\boldsymbol{p} \times \boldsymbol{q}$ and $\mu$ a partition with $m_{1}(\mu)=0$ and $k=|\mu| \leq|\lambda|=n$.

(i) The quantity $\vartheta_{\mu}^{\lambda}(\alpha)$ is a polynomial in the indeterminates $(\boldsymbol{p}, \boldsymbol{q})$ and $\beta=\alpha-1$, with integer coefficients.

(ii) The coefficients of the polynomial $(-1)^{k} \vartheta_{\mu}^{\lambda}(\alpha)$ in the indeterminates $(\boldsymbol{p},-\boldsymbol{q}, \beta)$ are nonnegative integers.

(iii) At least one of these coefficients is 1 .

Here two remarks are needed. Firstly, Stanley's ex-conjecture corresponds to the case $\alpha=1$ of our conjecture. This is a consequence of the Frobenius formula for the Schur functions $s_{\lambda}$. Actually we have (see [11, Examples 1.1.1 and 1.7.6] and [16, p. 78])

$$
s_{\lambda}=\sum_{\rho} z_{\rho}^{-1} \chi_{\rho}^{\lambda} p_{\rho} \quad \text { and } \quad J_{\lambda}(1)=\frac{n !}{\operatorname{dim} \lambda} s_{\lambda} .
$$

This yields

and for $\rho=\left(\mu, 1^{n-k}\right)$,

$$
z_{\rho} \theta_{\rho}^{\lambda}(1)=n ! \frac{\chi_{\rho}^{\lambda}}{\operatorname{dim} \lambda}
$$

$$
\widehat{\chi}_{\mu, 1^{n-k}}^{\lambda}=\left(\begin{array}{c}
n-k+m_{1}(\mu) \\
m_{1}(\mu)
\end{array}\right) \vartheta_{\mu}^{\lambda}(1) .
$$

Secondly, expanding the Jack polynomials in terms of the "augmented" monomial symmetric functions, which are integral combinations of the power sums [11, p.110], and using the results of [4], it is not difficult to see that $\vartheta_{\mu}^{\lambda}(\alpha)$ is a polynomial in $\alpha$, hence in $\beta$. It is also easy to prove that it is a polynomial in $(\boldsymbol{p}, \boldsymbol{q})$.

We have checked our conjecture for $m \leq 3$ and for any partition $\mu$ with $m_{1}(\mu)=0$ and $|\mu|-l(\mu) \leq 8$. These data are available on a web page [10]. For $m=2$ after substitution of $-\boldsymbol{q}$ to $\boldsymbol{q}$, the first values are as follows :

$$
\begin{aligned}
\vartheta_{2}^{\lambda}(\alpha)= & p_{1} q_{1}^{2}+p_{2} q_{2}^{2}+2 p_{1} p_{2} q_{2}+p_{1}^{2} q_{1}+p_{2}^{2} q_{2} \\
& +\beta\left(p_{1} q_{1}+p_{2} q_{2}+p_{1} q_{1}^{2}+p_{2} q_{2}^{2}\right) \\
-\vartheta_{3}^{\lambda}(\alpha)= & p_{1} q_{1}+p_{2} q_{2}+p_{1} q_{1}^{3}+p_{2} q_{2}^{3}+p_{1}^{3} q_{1}+p_{2}^{3} q_{2} \\
& +3 p_{1}^{2} p_{2} q_{2}+3 p_{1} p_{2}^{2} q_{2}+3 p_{1} p_{2} q_{2}^{2}+3 p_{1} p_{2} q_{1} q_{2}+3 p_{1}^{2} q_{1}^{2}+3 p_{2}^{2} q_{2}^{2} \\
& +\beta\left(p_{1} q_{1}+p_{2} q_{2}+3 p_{1} q_{1}^{2}+3 p_{2} q_{2}^{2}+3 p_{1}^{2} q_{1}+3 p_{2}^{2} q_{2}+6 p_{1} p_{2} q_{2}\right. \\
& \left.+3 p_{1}^{2} q_{1}^{2}+3 p_{2}^{2} q_{2}^{2}+3 p_{1} p_{2} q_{2}^{2}+2 p_{1} q_{1}^{3}+2 p_{2} q_{2}^{3}+3 p_{1} p_{2} q_{1} q_{2}\right) \\
& +\beta^{2}\left(2 p_{1} q_{1}+2 p_{2} q_{2}+3 p_{1} q_{1}^{2}+3 p_{2} q_{2}^{2}+p_{1} q_{1}^{3}+p_{2} q_{2}^{3}\right) .
\end{aligned}
$$


In this paper we present a general method to obtain linear identities between the coefficients $\theta_{\rho}^{\lambda}$. We apply this method in the simplest case $m=1$, i.e. for $\lambda=p \times q$, the rectangular shape formed by $p$ parts equal to $q$.

In this situation, we prove that $(-1)^{k} \vartheta_{\mu}^{\lambda}(\alpha)$ is a polynomial in $(p,-q, \beta)$, with nonnegative rational coefficients. The proof is much more cumbersome and lengthy than in the case $\alpha=1$, studied in $[17,15]$.

We use induction on the weight $|\mu|=k$. An explicit recurrence formula generates $(-1)^{k} \vartheta_{\mu}^{\lambda}(\alpha)$ as a polynomial in $(p,-q, \alpha, \beta)$, with nonnegative rational coefficients. However in spite of empirical evidence, we are in lack of an argument proving that these rational numbers are actually integers.

After substitution of $-q$ to $q$, the first values are

$$
\begin{aligned}
\vartheta_{2}^{\lambda}(\alpha)= & p q(\alpha q+p+\beta), \\
-\vartheta_{3}^{\lambda}(\alpha)= & p q(\alpha q+p+\beta)(\alpha q+p+2 \beta)+\alpha p q(p q+1), \\
\vartheta_{4}^{\lambda}(\alpha)= & p q((\alpha q+p+\beta)(\alpha q+p+2 \beta)+\alpha(p q+1))(\alpha q+p+3 \beta) \\
& +2 \alpha p q(p q+2)(\alpha q+p+\beta), \\
\vartheta_{22}^{\lambda}(\alpha)= & 2 p q(\alpha q+p+\beta)(\alpha q+p+2 \beta)+2 \alpha p q(p q+1) \\
& +p q(p q+2)(\alpha q+p+\beta)^{2} .
\end{aligned}
$$

We conjecture that such a property keeps true in the general case.

Conjecture 2. Let $\lambda=\boldsymbol{p} \times \boldsymbol{q}$ and $\mu$ a partition with $m_{1}(\mu)=0$ and $k=|\mu| \leq$ $|\lambda|=n$. The quantity $(-1)^{k} \vartheta_{\mu}^{\lambda}(\alpha)$ has some "natural" expression as a polynomial in $(\boldsymbol{p},-\boldsymbol{q}, \alpha, \beta)$ with nonnegative integer coefficients.

Actually our results suggest the existence of some mysterious $(\alpha, \beta)$-scheme, underlying the classical theory of Jack polynomials, where $\beta$ would play a role as important as $\alpha$. However we have no conjectured expression, nor any combinatorial interpretation, giving the quantity $(-1)^{k} \vartheta_{\mu}^{\lambda}(\alpha)$ as a polynomial in $(\alpha, \beta)$, with nonnegative integer coefficients.

The paper is organized as follows. In Section 2 we introduce our notations and recall general facts about (shifted) symmetric functions and (shifted) Jack polynomials. In Section 3, starting from the generalized binomial formula, we define an isomorphism between symmetric and shifted symmetric functions. In Sections 4 and 5 we use this method to obtain several linear identities between the $\theta_{\rho}^{\lambda}$. Section 6 is devoted to the case $m=1$. Finally Section 7 compares our proof with those previously given for $\alpha=1[17,15]$.

\section{2. (Shifted) Jack polynomials}

The standard reference for symmetric functions and Jack polynomials are [11, Section 6.10] and [16]. Although the theory of symmetric functions goes back to the early 19th century, the notion of "shifted symmetric" functions is quite recent. We refer to $[3,12,13,14]$ and to other references given there.

2.1. Symmetric functions. Let $x=\left\{x_{1}, x_{2}, x_{3}, \ldots\right\}$ be an infinite set of indeterminates, and $\mathcal{S}$ the corresponding algebra of symmetric functions with coefficients in $\mathbf{Q}$. Let $\mathbf{Q}[\alpha]$ be the field of rational functions in some indeterminate $\alpha$ (which may be considered as a positive real number), and $\mathbf{S}=\mathcal{S} \otimes \mathbf{Q}[\alpha]$ the algebra of symmetric 
functions with coefficients in $\mathbf{Q}[\alpha]$. The parameter $\alpha$ being kept fixed, for clarity of display, we shall omit its dependence in any notation below.

A partition $\lambda=\left(\lambda_{1}, \ldots, \lambda_{r}\right)$ is a finite weakly decreasing sequence of nonnegative integers, called parts. The number $l(\lambda)$ of positive parts is called the length of $\lambda$, and $|\lambda|=\sum_{i=1}^{r} \lambda_{i}$ the weight of $\lambda$. For any integer $i \geq 1, m_{i}(\lambda)=\operatorname{card}\left\{j: \lambda_{j}=i\right\}$ is the multiplicity of the part $i$ in $\lambda$. Clearly $l(\lambda)=\sum_{i} m_{i}(\lambda)$ and $|\lambda|=\sum_{i} i m_{i}(\lambda)$. We also write $\lambda=\left(1^{m_{1}(\lambda)}, 2^{m_{2}(\lambda)}, 3^{m_{3}(\lambda)}, \ldots\right)$ and set

$$
z_{\lambda}=\prod_{i \geq 1} i^{m_{i}(\lambda)} m_{i}(\lambda) !
$$

Being given two partitions, we write $\mu \subseteq \lambda$ if $\mu_{i} \leq \lambda_{i}$ for any $i$. We denote $\lambda^{\prime}$ the partition conjugate to $\lambda$, whose parts are given by $m_{i}\left(\lambda^{\prime}\right)=\lambda_{i}-\lambda_{i+1}$. We have $\lambda_{i}^{\prime}=\sum_{j \geq i} m_{j}(\lambda)$.

We define

$$
\begin{aligned}
h_{\lambda} & =\prod_{(i, j) \in \lambda}\left(\lambda_{j}^{\prime}-i+1+\alpha\left(\lambda_{i}-j\right)\right), \\
h_{\lambda}^{\prime} & =\prod_{(i, j) \in \lambda}\left(\lambda_{j}^{\prime}-i+\alpha\left(\lambda_{i}-j+1\right)\right), \\
(u)_{\lambda} & =\prod_{(i, j) \in \lambda}(u+j-1-(i-1) / \alpha) .
\end{aligned}
$$

The last quantity is a generalization of the "raising" factorial, in terms of the " $\alpha$ contents" $j-1-(i-1) / \alpha$.

The power sum symmetric functions are defined by $p_{k}(x)=\sum_{i \geq 1} x_{i}^{k}$. They form an algebraic basis of $\mathbf{S}$. A linear basis is given by the symmetric functions

$$
p_{\lambda}=\prod_{i=1}^{l(\lambda)} p_{\lambda_{i}}=\prod_{i \geq 1} p_{i}{ }^{m_{i}(\lambda)} .
$$

The algebra $\mathbf{S}$ may be endowed with a scalar product $<,>$ for which we have two orthogonal bases, both indexed by partitions :

(i) the basis of power sum symmetric functions, with

$$
<p_{\lambda}, p_{\mu}>=\delta_{\lambda \mu} \alpha^{l(\lambda)} z_{\lambda},
$$

(ii) the basis of (suitably normalized) Jack symmetric functions, with

$$
<J_{\lambda}, J_{\mu}>=\delta_{\lambda \mu} h_{\lambda} h_{\lambda}^{\prime} .
$$

We write $\theta_{\rho}^{\lambda}$ for the transition matrix between these two orthogonal bases, namely

$$
J_{\lambda}=\sum_{|\rho|=|\lambda|} \theta_{\rho}^{\lambda} p_{\rho} .
$$

If we restrict to a finite set of $N$ indeterminates $x=\left(x_{1}, \ldots, x_{N}\right)$, we have

$$
J_{\lambda}\left(1^{N}\right):=J_{\lambda}(1, \ldots, 1)=\alpha^{|\lambda|}(N / \alpha)_{\lambda} .
$$

Denoting $j_{\lambda}=h_{\lambda} h_{\lambda}^{\prime}$, we introduce

$$
J_{\lambda}^{\sharp}=\frac{J_{\lambda}}{j_{\lambda}}, \quad J_{\lambda}^{\star}=\frac{J_{\lambda}}{J_{\lambda}\left(1^{N}\right)} .
$$


The first relation defines the basis dual of $J_{\lambda}$ with respect to the scalar product $<,>$. In contrast with $J_{\lambda}^{\star}$, it does not depend on $N$. We have

$$
\prod_{i, j=1}^{N}\left(1-x_{i} y_{j}\right)^{-1 / \alpha}=\sum_{\lambda} J_{\lambda}(x) J_{\lambda}^{\sharp}(y) .
$$

2.2. Shifted symmetric functions. A polynomial in $N$ indeterminates $x=\left(x_{1}, \ldots, x_{N}\right)$ with coefficients in $\mathbf{Q}[\alpha]$ is said to be "shifted symmetric" if it is symmetric in the $N$ "shifted variables" $x_{i}-i / \alpha$.

Dealing with an infinite set of indeterminates $x=\left\{x_{1}, x_{2}, x_{3}, \ldots\right\}$, in analogy with symmetric functions, a "shifted symmetric function" $f$ is a family $\left\{f_{i}, i \geq 1\right\}$ with the two following properties :

(i) $f_{i}$ is shifted symmetric in $\left(x_{1}, x_{2}, \ldots, x_{i}\right)$,

(ii) $f_{i+1}\left(x_{1}, x_{2}, \ldots, x_{i}, 0\right)=f_{i}\left(x_{1}, x_{2}, \ldots, x_{i}\right)$.

This defines $\mathbf{S}^{*}$, the algebra of shifted symmetric functions with coefficients in $\mathbf{Q}[\alpha]$. A typical example is provided by the "shifted power sums"

$$
p_{k}^{\star}(x)=\sum_{i \geq 1}\left(\left[x_{i}-(i-1) / \alpha\right]_{k}-[-(i-1) / \alpha]_{k}\right),
$$

with $[x]_{k}=x(x-1) \cdots(x-k+1)$. These shifted symmetric functions generate $\mathbf{S}^{*}$ algebraically.

Any element $f \in \mathbf{S}^{*}$ may be evaluated at any sequence $x=\left(x_{1}, x_{2}, \ldots\right)$ with finitely many non zero terms, hence at any partition $\lambda$. Moreover by analyticity, $f$ is entirely determined by its restriction $f(\lambda)$ to partitions. This identification is usually performed and $\mathbf{S}^{*}$ is considered as a function algebra on the set of partitions.

For any partition $\mu$ there exists a shifted symmetric function $J_{\mu}^{\dagger}$ such that

(i) degree $J_{\mu}^{\dagger}=|\mu|$,

(ii) $J_{\mu}^{\dagger}(\lambda)=0$ except if $\mu \subseteq \lambda$, and $J_{\mu}^{\dagger}(\mu) \neq 0$.

It is a very remarkable fact that in this definition, the overdetermined system of linear conditions (ii) may be replaced by the weaker conditions

(iii) $J_{\mu}^{\dagger}(\lambda)=0$ except if $|\mu| \leq|\lambda|$, and $J_{\mu}^{\dagger}(\mu) \neq 0$.

The function $J_{\mu}^{\dagger}$ is called the "shifted Jack polynomial" associated with $\mu$. It is unique up to the value of $J_{\mu}^{\dagger}(\mu)$.

A map $\mathbf{S}^{*} \rightarrow \mathbf{S}$ can be defined, which associates to any shifted symmetric function $f \in \mathbf{S}^{*}$ its "leading symmetric term" denoted $[f]$. By definition $[f]$ is the highest degree term of $f$, which is necessarily symmetric.

It is another very remarkable fact that Jack polynomials are the leading symmetric terms of shifted Jack polynomials. More precisely we have

$$
\left[\frac{J_{\mu}^{\dagger}}{J_{\mu}^{\dagger}(\mu)}\right]=\alpha^{|\mu|} J_{\mu}^{\sharp} .
$$

Hence the family $\left\{J_{\mu}^{\dagger} / J_{\mu}^{\dagger}(\mu)\right\}$ forms a basis of the algebra $\mathbf{S}^{*}$. 
2.3. Generalized binomial formula. Jack polynomials allow us to write the following generalization of the classical binomial formula

$$
J_{\lambda}^{\star}\left(1+x_{1}, \ldots, 1+x_{N}\right)=\sum_{\mu \subseteq \lambda}\left(\begin{array}{c}
\lambda \\
\mu
\end{array}\right) J_{\mu}^{\star}\left(x_{1}, \ldots, x_{N}\right),
$$

which was first studied independently in $[2,6]$.

The generalized binomial coefficients thus introduced may be given the following alternative definition

$$
\exp \left(p_{1}\right) J_{\mu}^{\sharp}=\sum_{\lambda \supseteq \mu} \alpha^{|\lambda|-|\mu|}\left(\begin{array}{l}
\lambda \\
\mu
\end{array}\right) J_{\lambda}^{\sharp} .
$$

The equivalence of both properties was proved in [7], as the limit of a more general result, obtained in the framework of Macdonald polynomials. This second definition has the advantage of being independent of $N$.

It was first observed in [12] that the generalized binomial coefficient $\left(\begin{array}{l}\lambda \\ \mu\end{array}\right)$ is merely the shifted Jack polynomial $J_{\mu}^{\dagger}(\lambda)$ suitably normalized:

$$
\frac{J_{\mu}^{\dagger}(\lambda)}{J_{\mu}^{\dagger}(\mu)}=\left(\begin{array}{c}
\lambda \\
\mu
\end{array}\right)
$$

This property is actually a special case of a more general correspondence, that we shall make explicit in the next section.

\section{Symmetric vs shifted symmetric}

Given any symmetric function $f \in \mathbf{S}$, we write

$$
\exp \left(p_{1}\right) f=\sum_{\lambda} \alpha^{|\lambda|} f^{\#}(\lambda) J_{\lambda}^{\sharp},
$$

i.e. we develop the inhomogeneous symmetric left-hand side in terms of the Jack polynomials basis.

Then the results recalled in Section 2 can be rephrased as follows.

Theorem. The coefficient $f^{\#}(\lambda)$ is a shifted symmetric function of $\lambda$. The map $f \rightarrow f^{\#}$ is an isomorphism of $\mathbf{S}$ onto $\mathbf{S}^{*}$. If $f$ is homogeneous, one has $\left[f^{\#}\right]=f$.

A direct proof would be possible, but it is out of the scope of this paper. Here we shall only mention that relations (2.3) and (2.4) imply

$$
\left(\alpha^{|\mu|} J_{\mu}^{\sharp}\right)^{\#}=\frac{J_{\mu}^{\dagger}}{J_{\mu}^{\dagger}(\mu)},
$$

from which follows

$$
f^{\#}=\sum_{\mu} \alpha^{-|\mu|}<f, J_{\mu}>\frac{J_{\mu}^{\dagger}}{J_{\mu}^{\dagger}(\mu)} .
$$

Observe that if $f$ is not homogeneous, $\left[f^{\#}\right]$ is its highest degree term.

We now give some examples and properties of this isomorphism. 
Proposition 1. Let $f \in \mathbf{S}$ be a symmetric function, homogeneous of degree $k$. For any positive integer $r$, we have

$$
\begin{aligned}
& \text { (i) }\left(\frac{p_{1}^{r}}{r !} f\right)^{\#}(\lambda)=\left(\begin{array}{c}
|\lambda|-k \\
r
\end{array}\right) f^{\#}(\lambda), \\
& \text { (ii) }\left(\begin{array}{c}
|\lambda|-k \\
r-k
\end{array}\right) f^{\#}(\lambda)=\sum_{|\rho|=r}\left(\begin{array}{l}
\lambda \\
\rho
\end{array}\right) f^{\#}(\rho) .
\end{aligned}
$$

Proof. By the definition (3.1) for any $s \geq 0$ we have

$$
\frac{p_{1}^{s}}{s !} f=\sum_{|\lambda|=k+s} \alpha^{|\lambda|} f^{\#}(\lambda) J_{\lambda}^{\sharp} .
$$

Hence

$$
\exp \left(p_{1}\right) \frac{p_{1}^{r}}{r !} f=\sum_{s \geq 0} \sum_{|\lambda|=k+r+s} \frac{(r+s) !}{r ! s !} \alpha^{|\lambda|} f^{\#}(\lambda) J_{\lambda}^{\sharp},
$$

and (i). On the other hand, we have

$$
\begin{aligned}
\exp \left(p_{1}\right) \frac{p_{1}^{r}}{r !} f & =\sum_{\rho} \alpha^{|\rho|} f^{\#}(\rho) \frac{p_{1}^{r}}{r !} J_{\rho}^{\sharp} \\
& =\sum_{\rho} \alpha^{|\rho|} f^{\#}(\rho) \sum_{|\lambda|=|\rho|+r} \alpha^{r}\left(\begin{array}{c}
\lambda \\
\rho
\end{array}\right) J_{\lambda}^{\sharp},
\end{aligned}
$$

where the second equality is a straightforward consequence of (2.3). In other words,

$$
\left(\frac{p_{1}^{r}}{r !} f\right)^{\#}(\lambda)=\sum_{|\rho|=|\lambda|-r}\left(\begin{array}{l}
\lambda \\
\rho
\end{array}\right) f^{\#}(\rho) .
$$

Comparing with (i), we obtain (ii).

In particular writing (ii) with $f=\alpha^{|\mu|} J_{\mu}^{\sharp}$ yields

$$
\left(\begin{array}{c}
|\lambda|-|\mu| \\
r-|\mu|
\end{array}\right)\left(\begin{array}{l}
\lambda \\
\mu
\end{array}\right)=\sum_{|\rho|=r}\left(\begin{array}{l}
\lambda \\
\rho
\end{array}\right)\left(\begin{array}{l}
\rho \\
\mu
\end{array}\right) .
$$

We are interested in the isomorphism $f \rightarrow f^{\#}$ because of the following important example.

Proposition 2. Let $\mu$ be a partition with weight $|\mu|=k$. For any partition $\lambda$ with $|\lambda|=n \geq k$, we have

$$
\alpha^{k-l(\mu)}\left(p_{\mu}\right)^{\#}(\lambda)=\left(\begin{array}{c}
n-k+m_{1}(\mu) \\
m_{1}(\mu)
\end{array}\right) z_{\mu} \theta_{\mu, 1^{n-k}}^{\lambda} .
$$

Thus $\vartheta_{\mu}^{\lambda}=z_{\mu} \theta_{\mu, 1^{n-k}}^{\lambda}$ is a shifted symmetric function of $\lambda$. 
Proof. For any partition $\rho$, by orthogonality of the power sums we have

$$
<J_{\lambda}, p_{\rho}>=\alpha^{l(\rho)} z_{\rho} \theta_{\rho}^{\lambda} .
$$

Hence by orthogonality of the Jack polynomials,

$$
p_{\rho}=\alpha^{l(\rho)} z_{\rho} \sum_{|\lambda|=n} \theta_{\rho}^{\lambda} J_{\lambda}^{\sharp} .
$$

By the definition (3.1) for $\rho=\left(\mu, 1^{n-k}\right)$ this implies

$$
\left(p_{\mu}\right)^{\#}(\lambda)=\alpha^{l(\mu)-k} \frac{z_{\mu, 1^{n-k}}}{(n-k) !} \theta_{\mu, 1^{n-k}}^{\lambda} .
$$

Corollary. Let $\mu$ be a partition with weight $|\mu|=k$. For any partition $\lambda$ with $|\lambda|=$ $n \geq k$ and any $r \geq 0$, we have

$$
\left(\begin{array}{c}
n-k+m_{1}(\mu) \\
n-r
\end{array}\right) \theta_{\mu, 1^{n-k}}^{\lambda}=\sum_{|\rho|=r}\left(\begin{array}{l}
\lambda \\
\rho
\end{array}\right) \theta_{\mu, 1^{r-k}}^{\rho} .
$$

Proof. Follows from Proposition 1 (ii) written for $f=\alpha^{k-l(\mu)} p_{\mu}$.

Observe that for $\lambda=\boldsymbol{p} \times \boldsymbol{q}$, Proposition 2 implies that $\vartheta_{\mu}^{\lambda}$ is a polynomial in $(\boldsymbol{p}, \boldsymbol{q})$ with coefficients in $\mathbf{Q}[\alpha]$, since it is a shifted symmetric function of $\lambda=\boldsymbol{p} \times \boldsymbol{q}$.

For $\alpha=1$ Proposition 2 is connected with Stanley's ex-conjecture. Indeed equation (1.1) writes as

$$
\widehat{\chi}_{\mu, 1^{n-k}}^{\lambda}=\left(\begin{array}{c}
n-k+m_{1}(\mu) \\
m_{1}(\mu)
\end{array}\right) \vartheta_{\mu}^{\lambda}=\left(p_{\mu}\right)^{\#}(\lambda) .
$$

Therefore it might seem better to remove the restriction $m_{1}(\mu)=0$ in our conjectures. Instead of $\vartheta_{\mu}^{\lambda}$, they should be written for the quantities

$$
\left(\begin{array}{c}
n-k+m_{1}(\mu) \\
m_{1}(\mu)
\end{array}\right) \vartheta_{\mu}^{\lambda}=\alpha^{k-l(\mu)}\left(p_{\mu}\right)^{\#}(\lambda) .
$$

However this extension would only bring a trivial factor. Actually for $m_{1}(\mu) \neq 0$, if we denote by $\tilde{\mu}$ the partition obtained by removing the parts 1 of $\mu$, we have obviously $\theta_{\mu, 1^{n-k}}^{\lambda}=\theta_{\tilde{\mu}, 1^{n-k+m_{1}(\mu)}}^{\lambda}$. Hence $\vartheta_{\mu}^{\lambda}=m_{1}(\mu) ! \vartheta_{\tilde{\mu}}^{\lambda}$ and

$$
\left(\begin{array}{c}
n-k+m_{1}(\mu) \\
m_{1}(\mu)
\end{array}\right) \vartheta_{\mu}^{\lambda}=(n-k+1) \cdots\left(n-k+m_{1}(\mu)\right) \vartheta_{\tilde{\mu}}^{\lambda} .
$$

Since $n=\sum_{i=1}^{m} p_{i} q_{i}$, the integrality and positivity properties of the first product are obvious. Therefore there is no loss of generality in restricting to the study of $\vartheta_{\tilde{\mu}}^{\lambda}$. 


\section{Linear identities}

4.1. Our method. Let $C$ and $D$ be two linear operators acting on $\mathbf{S}$. We denote by $\{D, C\}^{n}$ the $n$-th nested commutator

$$
\{D, C\}^{n}=[[\cdots[D, C] \cdots, C], C],
$$

with $\{D, C\}^{0}=D$ and $\{D, C\}^{1}=[D, C]=D C-C D$. Then we have the classical formula (often misnamed as the Baker-Campbell-Hausdorff formula)

$$
e^{-t C} D e^{t C}=\sum_{n \geq 0} \frac{t^{n}}{n !}\{D, C\}^{n} .
$$

This formula is an easy consequence of the following property

$$
\{D, C\}^{n}=\sum_{r=0}^{n}(-1)^{n-r}\left(\begin{array}{l}
n \\
r
\end{array}\right) C^{n-r} D C^{r},
$$

which is immediately proved by induction. Therefore, denoting also by $p_{k}$ the multiplication operator acting on $\mathbf{S}$ by $f \rightarrow p_{k} f$, we have

$$
D\left(\exp \left(p_{1}\right) f\right)=\exp \left(p_{1}\right)\left(\sum_{n \geq 0} \frac{1}{n !}\left\{D, p_{1}\right\}^{n} f\right) .
$$

Let us assume that the action of $D$ on Jack polynomials is explicitly known, i.e.

$$
D J_{\rho}^{\sharp}=\sum_{\sigma} a_{\rho \sigma} J_{\sigma}^{\sharp} .
$$

We may apply $D$ to (3.1), which yields

$$
D\left(\exp \left(p_{1}\right) f\right)=\sum_{\lambda} \alpha^{|\lambda|} f^{\#}(\lambda) D J_{\lambda}^{\sharp}=\sum_{\lambda, \rho} \alpha^{|\lambda|} f^{\#}(\lambda) a_{\lambda \rho} J_{\rho}^{\sharp} .
$$

By comparison with (4.1), we obtain the following linear identity between shifted symmetric functions

$$
\sum_{n \geq 0} \frac{1}{n !}\left(\left\{D, p_{1}\right\}^{n} f\right)^{\#}(\lambda)=\sum_{\rho} \alpha^{|\rho|-|\lambda|} a_{\rho \lambda} f^{\#}(\rho) .
$$

If moreover the action of $D$ is explicitly known on the power sums, by specializing $f=$ $\alpha^{|\mu|-l(\mu)} p_{\mu}$ and using (3.2), we shall obtain a linear identity between some coefficients $\theta_{\rho}^{\lambda}$. Many examples are given below.

4.2. Pieri formula. For any partition $\lambda$ and any integer $1 \leq i \leq l(\lambda)+1$, we denote by $\lambda^{(i)}$ the partition $\mu$ (if it exists) such that $\mu_{j}=\lambda_{j}$ for $j \neq i$ and $\mu_{i}=\lambda_{i}+1$. Similarly for any integer $1 \leq i \leq l(\lambda)$, we denote by $\lambda_{(i)}$ the partition $\nu$ (if it exists) such that $\nu_{j}=\lambda_{j}$ for $j \neq i$ and $\nu_{i}=\lambda_{i}-1$.

Jack polynomials satisfy the following generalization of Pieri formula $[11,16]$ :

$$
p_{1} J_{\lambda}=\sum_{i=1}^{l(\lambda)+1} c_{i}(\lambda) J_{\lambda^{(i)}} .
$$


The Pieri coefficients $c_{i}(\lambda)$ have the following analytic expression [5], see also [7, Prop. 5, p.299]:

$$
c_{i}(\lambda)=\frac{1}{\alpha \lambda_{i}+l(\lambda)-i+2} \prod_{\substack{j=1 \\ j \neq i}}^{l(\lambda)+1} \frac{\alpha\left(\lambda_{i}-\lambda_{j}\right)+j-i+1}{\alpha\left(\lambda_{i}-\lambda_{j}\right)+j-i} .
$$

In $[7$, p.300] (see also [6]) we have proved that these coefficients are connected with the generalized binomial coefficients by

$$
c_{i}(\lambda)=\alpha\left(\begin{array}{c}
\lambda^{(i)} \\
\lambda
\end{array}\right) \frac{j_{\lambda}}{j_{\lambda^{(i)}}}, \quad \alpha\left(\begin{array}{c}
\lambda \\
\lambda_{(i)}
\end{array}\right)=c_{i}\left(\lambda_{(i)}\right) \frac{j_{\lambda}}{j_{\lambda_{(i)}}},
$$

and that we have

$$
\alpha\left(\begin{array}{c}
\lambda \\
\lambda_{(i)}
\end{array}\right)=\left(\alpha \lambda_{i}+l(\lambda)-i\right) \prod_{\substack{j=1 \\
j \neq i}}^{l(\lambda)} \frac{\alpha\left(\lambda_{i}-\lambda_{j}\right)+j-i-1}{\alpha\left(\lambda_{i}-\lambda_{j}\right)+j-i} .
$$

The method of Section 4.1 is very easy to apply for $D=p_{1}$ or $D=\partial / \partial p_{1}$, since in these cases we have $\left[D, p_{1}\right]=0$ or 1 , respectively. We write $D^{\perp}$ for the adjoint of any linear operator $D$ with respect to the scalar product $<,>$.

Proposition 3. For any symmetric function $f \in \mathbf{S}$ we have

$$
\begin{aligned}
& \text { (i) }\left(p_{1} f\right)^{\#}(\lambda)=\sum_{i=1}^{l(\lambda)}\left(\begin{array}{c}
\lambda \\
\lambda_{(i)}
\end{array}\right) f^{\#}\left(\lambda_{(i)}\right), \\
& \text { (ii) }\left(\frac{\partial}{\partial p_{1}} f\right)^{\#}(\lambda)+f^{\#}(\lambda)=\sum_{i=1}^{l(\lambda)+1} c_{i}(\lambda) f^{\#}\left(\lambda^{(i)}\right) .
\end{aligned}
$$

If $f$ is homogeneous of degree $k$, (i) is equal to $(|\lambda|-k) f^{\#}(\lambda)$.

Proof. To prove (i) we may apply $p_{1}$ to (3.1), which yields

$$
\begin{aligned}
p_{1} \exp \left(p_{1}\right) f & =\sum_{\lambda} \alpha^{|\lambda|} f^{\#}(\lambda) \sum_{i=1}^{l(\lambda)+1} c_{i}(\lambda) \frac{j_{\lambda^{(i)}}}{j_{\lambda}} J_{\lambda^{(i)}}^{\sharp} \\
& =\sum_{\mu} \alpha^{|\mu|-1} J_{\mu}^{\sharp} \sum_{i=1}^{l(\mu)} f^{\#}\left(\mu_{(i)}\right) c_{i}\left(\mu_{(i)}\right) \frac{j_{\mu}}{j_{\mu_{(i)}}},
\end{aligned}
$$

where the second equality is obtained by setting $\mu=\lambda^{(i)}$. Using (4.2), we get

$$
\exp \left(p_{1}\right) p_{1} f=\sum_{\mu} \alpha^{|\mu|} J_{\mu}^{\sharp} \sum_{i=1}^{l(\mu)}\left(\begin{array}{c}
\mu \\
\mu_{(i)}
\end{array}\right) f^{\#}\left(\mu_{(i)}\right) .
$$

To prove (ii) observe that $p_{1}^{\perp}=\alpha \partial / \partial p_{1}$. This can be proved by the same argument as in $[11$, p.76]: by linearity, it is enough to check the fact on power sums. But in $[7$, Prop. 11, p.306] we have shown that

$$
p_{1}^{\perp} J_{\lambda}=\alpha \sum_{i=1}^{l(\lambda)}\left(\begin{array}{c}
\lambda \\
\lambda_{(i)}
\end{array}\right) J_{\lambda_{(i)}} .
$$


Applying $\partial / \partial p_{1}$ to (3.1) we thus obtain

$$
\begin{aligned}
\frac{\partial}{\partial p_{1}}\left(\exp \left(p_{1}\right) f\right) & =\exp \left(p_{1}\right) \frac{\partial}{\partial p_{1}} f+\exp \left(p_{1}\right) f \\
& =\sum_{\lambda} \alpha^{|\lambda|} f^{\#}(\lambda) \sum_{i=1}^{l(\lambda)}\left(\begin{array}{c}
\lambda \\
\lambda_{(i)}
\end{array}\right) \frac{j_{\lambda_{(i)}}}{j_{\lambda}} J_{\lambda_{(i)}}^{\sharp} \\
& =\sum_{\mu} \alpha^{|\mu|+1} J_{\mu}^{\sharp} \sum_{i=1}^{l(\mu)+1}\left(\begin{array}{c}
\mu^{(i)} \\
\mu
\end{array}\right) \frac{j_{\mu}}{j_{\mu^{(i)}}} f^{\#}\left(\mu^{(i)}\right) \\
& =\sum_{\mu} \alpha^{|\mu|} J_{\mu}^{\sharp} \sum_{i=1}^{l(\mu)+1} c_{i}(\mu) f^{\#}\left(\mu^{(i)}\right) .
\end{aligned}
$$

Again the last equalities are obtained by setting $\mu=\lambda_{(i)}$ and applying (4.2).

The same argument, a change $\mu=\lambda_{(i)}$ or $\mu=\lambda^{(i)}$ followed by use of (4.2), will be tacitly used in all proofs of this section. Another elementary application of our method is given by the differential operator $E_{0}=\sum_{i=1}^{N} \partial / \partial x_{i}$, which is dependent on the number of variables $N$.

Proposition 4. For any symmetric function $f \in \mathbf{S}$ we have

$$
\left(E_{0} f\right)^{\#}(\lambda)+N f^{\#}(\lambda)=\sum_{i=1}^{l(\lambda)+1} c_{i}(\lambda)\left(N+\alpha \lambda_{i}-i+1\right) f^{\#}\left(\lambda^{(i)}\right) .
$$

Proof. Applying $E_{0}$ to the generalized binomial formula (2.2), we obtain easily

$$
E_{0} J_{\lambda}^{\star}=\sum_{i=1}^{l(\lambda)}\left(\begin{array}{c}
\lambda \\
\lambda_{(i)}
\end{array}\right) J_{\lambda_{(i)}}^{\star} .
$$

Hence

$$
\begin{aligned}
E_{0}\left(\exp \left(p_{1}\right) f\right) & =\exp \left(p_{1}\right) E_{0} f+N \exp \left(p_{1}\right) f \\
& =\sum_{\lambda} \alpha^{|\lambda|} f^{\#}(\lambda) \sum_{i=1}^{l(\lambda)}\left(\begin{array}{c}
\lambda \\
\lambda_{(i)}
\end{array}\right) \frac{j_{\lambda_{(i)}}}{j_{\lambda}} \frac{J_{\lambda}\left(1^{N}\right)}{J_{\lambda_{(i)}}\left(1^{N}\right)} J_{\lambda_{(i)}}^{\sharp} \\
& =\sum_{\lambda} \alpha^{|\lambda|} J_{\lambda}^{\sharp} \sum_{i=1}^{l(\lambda)+1} c_{i}(\lambda) \frac{J_{\lambda^{(i)}}\left(1^{N}\right)}{J_{\lambda}\left(1^{N}\right)} f^{\#}\left(\lambda^{(i)}\right) .
\end{aligned}
$$

By (2.1) we have $J_{\lambda^{(i)}}\left(1^{N}\right) / J_{\lambda}\left(1^{N}\right)=N+\alpha \lambda_{i}-i+1$.

4.3. Other examples. For any integer $k \geq 0$ we introduce the differential operators

$$
\begin{aligned}
E_{k} & =\sum_{i=1}^{N} x_{i}^{k} \frac{\partial}{\partial x_{i}} \\
D_{k} & =\sum_{i=1}^{N} x_{i}^{k} \frac{\partial^{2}}{\partial x_{i}^{2}}+\frac{2}{\alpha} \sum_{\substack{i, j=1 \\
i \neq j}}^{N} \frac{x_{i}^{k}}{x_{i}-x_{j}} \frac{\partial}{\partial x_{i}} .
\end{aligned}
$$


For $k \neq 0, E_{k}$ is independent of $N$. It is not difficult to check that

$$
\begin{aligned}
D_{0} & =\left[E_{0}, D_{1}\right] \\
D_{1} & =\frac{1}{2}\left[E_{0}, D_{2}\right] \\
E_{2} & =\sum_{k \geq 1} k p_{k+1} \frac{\partial}{\partial p_{k}}=\frac{1}{2}\left[D_{2}, p_{1}\right]-\frac{1}{\alpha}(N-1) p_{1}, \\
E_{2}^{\perp} & =\sum_{k \geq 1}(k+1) p_{k} \frac{\partial}{\partial p_{k+1}}=E_{0}-N \frac{\partial}{\partial p_{1}} .
\end{aligned}
$$

If we write

$$
d_{1}(\lambda)=\sum_{(i, j) \in \lambda}(j-1-(i-1) / \alpha)
$$

for the sum of the $\alpha$-contents of $\lambda$, it is well known [16, p.84] that the Jack polynomials are eigenfunctions of $D_{2}$, namely

$$
D_{2} J_{\lambda}=2\left(d_{1}(\lambda)+|\lambda|(N-1) / \alpha\right) J_{\lambda}
$$

This implies

$$
\begin{aligned}
D_{1} J_{\lambda}^{\star} & =\sum_{i=1}^{l(\lambda)}\left(\begin{array}{c}
\lambda \\
\lambda_{(i)}
\end{array}\right)\left(\lambda_{i}-1+(N-i) / \alpha\right) J_{\lambda_{(i)}}^{\star}, \\
E_{2} J_{\lambda} & =\sum_{i=1}^{l(\lambda)+1} c_{i}(\lambda)\left(\lambda_{i}-(i-1) / \alpha\right) J_{\lambda^{(i)}}, \\
E_{2}^{\perp} J_{\lambda} & =\alpha \sum_{i=1}^{l(\lambda)}\left(\begin{array}{c}
\lambda \\
\lambda_{(i)}
\end{array}\right)\left(\lambda_{i}-1-(i-1) / \alpha\right) J_{\lambda_{(i)}} .
\end{aligned}
$$

We now apply the method of Section 4.1 to $E_{2}$ and $D_{1}$.

Proposition 5. For any symmetric function $f \in \mathbf{S}$ we have

$$
\left(E_{2} f\right)^{\#}(\lambda)+\left(p_{2} f\right)^{\#}(\lambda)=\sum_{i=1}^{l(\lambda)}\left(\begin{array}{c}
\lambda \\
\lambda_{(i)}
\end{array}\right)\left(\lambda_{i}-1-(i-1) / \alpha\right) f^{\#}\left(\lambda_{(i)}\right) .
$$

Proof. Using $\left[E_{2}, p_{1}\right]=p_{2}$, we have

$$
\begin{aligned}
E_{2}\left(\exp \left(p_{1}\right) f\right) & =\exp \left(p_{1}\right)\left(E_{2} f+p_{2} f\right) \\
& =\sum_{\lambda} \alpha^{|\lambda|} f^{\#}(\lambda) \sum_{i=1}^{l(\lambda)+1} c_{i}(\lambda)\left(\lambda_{i}-(i-1) / \alpha\right) \frac{j_{\lambda^{(i)}}}{j_{\lambda}} J_{\lambda^{(i)}}^{\sharp} \\
& =\sum_{\lambda} \alpha^{|\lambda|} J_{\lambda}^{\sharp} \sum_{i=1}^{l(\lambda)}\left(\begin{array}{c}
\lambda \\
\lambda_{(i)}
\end{array}\right)\left(\lambda_{i}-1-(i-1) / \alpha\right) f^{\#}\left(\lambda_{(i)}\right) .
\end{aligned}
$$


Proposition 6. For any symmetric function $f \in \mathbf{S}$ we have

$$
\begin{aligned}
& \left(\left(D_{1}+2 E_{1}+p_{1}+N(N-1) / \alpha\right) f\right)^{\#}(\lambda)= \\
& \alpha \sum_{i=1}^{l(\lambda)+1} c_{i}(\lambda)\left(\lambda_{i}+(N-i) / \alpha\right)\left(\lambda_{i}+(N-i+1) / \alpha\right) f^{\#}\left(\lambda^{(i)}\right) .
\end{aligned}
$$

Proof. We use $\left[D_{1}, p_{1}\right]=2 E_{1}+N(N-1) / \alpha$, hence $\left[\left[D_{1}, p_{1}\right], p_{1}\right]=2 p_{1}$. We have

$$
\begin{aligned}
D_{1}\left(\exp \left(p_{1}\right) f\right) & =\exp \left(p_{1}\right)\left(D_{1} f+2 E_{1} f+p_{1} f+N(N-1) f / \alpha\right) \\
& =\sum_{\lambda} \alpha^{|\lambda|} f^{\#}(\lambda) \sum_{i=1}^{l(\lambda)}\left(\begin{array}{c}
\lambda \\
\lambda_{(i)}
\end{array}\right)\left(\lambda_{i}-1+(N-i) / \alpha\right) \frac{J_{\lambda}\left(1^{N}\right)}{J_{\lambda_{(i)}}\left(1^{N}\right)} \frac{j_{\lambda_{(i)}}}{j_{\lambda}} J_{\lambda_{(i)}}^{\sharp} \\
& =\sum_{\lambda} \alpha^{|\lambda|} J_{\lambda}^{\sharp} \sum_{i=1}^{l(\lambda)+1} c_{i}(\lambda)\left(\lambda_{i}+(N-i) / \alpha\right) \frac{J_{\lambda^{(i)}}\left(1^{N}\right)}{J_{\lambda}\left(1^{N}\right)} f^{\#}\left(\lambda^{(i)}\right) .
\end{aligned}
$$

4.4. Generalization. The previous results may be extended as follows. Let $\Delta_{0}=$ $p_{1}, \nabla_{0}=E_{0}$ and for any $k>0$ define

$$
\Delta_{k}=\frac{1}{2}\left[D_{2}, \Delta_{k-1}\right], \quad \nabla_{k}=\frac{1}{2}\left[\nabla_{k-1}, D_{2}\right] .
$$

Clearly we have

$$
\Delta_{1}=E_{2}+(N-1) p_{1} / \alpha, \quad \nabla_{1}=D_{1} .
$$

Both families are in correspondence through $D \rightarrow D^{\perp}$. Starting from

$$
E_{0}^{\perp}=E_{2}+N p_{1} / \alpha
$$

we readily obtain

$$
\nabla_{k}^{\perp}=\Delta_{k+1}+\Delta_{k} / \alpha
$$

The operators $\Delta_{k}$ and $\nabla_{k}$ are respectively increasing and decreasing degree by 1 . We have

$$
\begin{aligned}
\Delta_{k} J_{\lambda} & =\sum_{i=1}^{l(\lambda)+1} c_{i}(\lambda)\left(\lambda_{i}+(N-i) / \alpha\right)^{k} J_{\lambda^{(i)}}, \\
\nabla_{k} J_{\lambda}^{\star} & =\sum_{i=1}^{l(\lambda)}\left(\begin{array}{c}
\lambda \\
\lambda_{(i)}
\end{array}\right)\left(\lambda_{i}-1+(N-i) / \alpha\right)^{k} J_{\lambda_{(i)}^{\star}}^{\star},
\end{aligned}
$$

which are easy consequences of $d_{1}\left(\lambda^{(i)}\right)-d_{1}(\lambda)=\lambda_{i}-(i-1) / \alpha$.

In this section we have applied our method to the operators $\Delta_{k}$ and $\nabla_{k}$ with $k=0,1$. The case $k=2$ is also easy to handle. For instance, we have

$$
\begin{aligned}
& \Delta_{2}=D_{3}+E_{2}+(N-1)^{2} p_{1} / \alpha^{2}, \\
& {\left[\Delta_{2}, p_{1}\right]=2 E_{3}+(2 N-3) p_{2} / \alpha+p_{1}^{2} / \alpha+p_{2},} \\
& {\left[\left[\Delta_{2}, p_{1}\right], p_{1}\right]=2 p_{3} .}
\end{aligned}
$$

For higher values of $k$, the computations become very messy. 
The operator $D_{2}$ is degree preserving and must be treated separately. Defining

$$
D_{2}^{\dagger}=D_{2}-2(N-1) E_{1} / \alpha
$$

we have obviously $D_{2}^{\dagger} J_{\lambda}=2 d_{1}(\lambda) J_{\lambda}$. Hence $D_{2}^{\dagger}$ is independent of $N$.

Proposition 7. For any symmetric function $f \in \mathbf{S}$ we have

$$
\left(\left(D_{2}^{\dagger}+2 E_{2}+p_{2}\right) f\right)^{\#}(\lambda)=2 d_{1}(\lambda) f^{\#}(\lambda)
$$

Proof. Consequence of $\left[D_{2}^{\dagger}, p_{1}\right]=2 E_{2}$ and $\left[\left[D_{2}^{\dagger}, p_{1}\right], p_{1}\right]=2 p_{2}$.

For completeness, we mention that using $p_{2}=\left[E_{2}, p_{1}\right]$ and $D_{0}=\left[E_{0}, D_{1}\right]$, the operators $p_{2}, D_{0}$, and $p_{2}^{\perp}=\alpha^{2} \partial / \partial p_{2}$ (which are respectively increasing and decreasing degree by 2) can also be managed very easily. This is left to the reader.

\section{Specialization to power sums}

We now specialize the results of Section 4 for $f=p_{\mu}$ with $|\mu|=k$ and $m_{1}(\mu)=0$. Then Proposition 2 reads

$$
\left(p_{\mu}\right)^{\#}(\lambda)=\alpha^{l(\mu)-k} \vartheta_{\mu}^{\lambda} .
$$

5.1. A lemma. The following result is proved by an easy induction on $N$.

Lemma. For any integer $r \geq 2$, we have

$$
2 \sum_{\substack{i, j=1 \\ i \neq j}}^{N} \frac{x_{i}^{r}}{x_{i}-x_{j}}=\sum_{i=1}^{r-2} p_{i} p_{r-i-1}+(2 N-r) p_{r-1} .
$$

By explicit computation we obtain a corollary which will be central for our purpose.

Proposition 8. Denote $\beta=\alpha-1$. For any integer $j \geq 0$, we have

$$
\begin{aligned}
D_{j} p_{\mu}=p_{\mu}( & \sum_{r, s} r s m_{r}(\mu)\left(m_{s}(\mu)-\delta_{r s}\right) \frac{p_{r+s+j-2}}{p_{r} p_{s}}+\frac{2 N-j}{\alpha} \sum_{r} r m_{r}(\mu) \frac{p_{r+j-2}}{p_{r}} \\
& \left.+\frac{\beta}{\alpha} \sum_{r} r(r-1) m_{r}(\mu) \frac{p_{r+j-2}}{p_{r}}+\frac{1}{\alpha} \sum_{r} r m_{r}(\mu) \sum_{i=1}^{r+j-3} \frac{p_{i} p_{r-i+j-2}}{p_{r}}\right) .
\end{aligned}
$$

For clarity of display, the following notations will be useful. We write $\mu \cup(s)$ when adding a part $s$ to a partition $\mu$, and $\mu \backslash(s)$ when substracting $s$. For any integer $r \geq 2$ we define (if they exist)

$$
\mu_{\downarrow(r)}=\mu \backslash(r) \cup(r-1), \quad \mu_{\uparrow(r)}=\mu \backslash(r) \cup(r+1) .
$$

We have $\left|\mu_{\downarrow(r)}\right|=k-1,\left|\mu_{\uparrow(r)}\right|=k+1, l\left(\mu_{\downarrow}(r)\right)=l\left(\mu_{\uparrow(r)}\right)=l(\mu)$.

Let $|\lambda|=n$. Recall that if $\nu=\mu \cup(1)$ is a partition having one part 1, Proposition 1 (i) yields

$$
\left(p_{\nu}\right)^{\#}(\lambda)=(n-|\mu|)\left(p_{\mu}\right)^{\#}(\lambda) .
$$

And if $\nu=\mu \cup(1,1)$ has two parts 1 , we have

$$
\left(p_{\nu}\right)^{\#}(\lambda)=(n-|\mu|)(n-|\mu|-1)\left(p_{\mu}\right)^{\#}(\lambda) .
$$


5.2. Raising degree. We first consider the cases of operators raising degree, i.e. $p_{1}$ and $E_{2}$. Proposition 3 (i) implies

$$
(n-k) \vartheta_{\mu}^{\lambda}=\sum_{i=1}^{l(\lambda)}\left(\begin{array}{c}
\lambda \\
\lambda_{(i)}
\end{array}\right) \vartheta_{\mu}^{\lambda_{(i)}} .
$$

Observe that it is merely (3.3) written for $r=n-1$.

An easy computation gives

$$
E_{2} p_{\mu}=\sum_{r} r m_{r}(\mu) p_{\mu_{\uparrow(r)}}
$$

so that Proposition 5 yields

$$
\vartheta_{\mu, 2}^{\lambda}+\sum_{r} r m_{r}(\mu) \vartheta_{\mu_{\uparrow(r)}}^{\lambda}=\alpha \sum_{i=1}^{l(\lambda)}\left(\begin{array}{c}
\lambda \\
\lambda_{(i)}
\end{array}\right)\left(\lambda_{i}-1-(i-1) / \alpha\right) \vartheta_{\mu}^{\lambda_{(i)}} .
$$

5.3. The operator $E_{0}$. We have easily

$$
E_{0} p_{\mu}=\sum_{r} r m_{r}(\mu) p_{\mu_{\downarrow}(r)} .
$$

Specializing Proposition 4, and identifying coefficients of $N$ on both sides of the identity, we get

$$
\vartheta_{\mu}^{\lambda}=\sum_{i=1}^{l(\lambda)+1} c_{i}(\lambda) \vartheta_{\mu}^{\lambda^{(i)}}
$$

which may be also obtained from Proposition 3 (ii).

If we identify terms in $N^{0}$, we obtain

$$
\sum_{r}^{\star} r m_{r}(\mu) \vartheta_{\mu_{\downarrow}(r)}^{\lambda}=\sum_{i=1}^{l(\lambda)+1} c_{i}(\lambda)\left(\lambda_{i}-(i-1) / \alpha\right) \vartheta_{\mu}^{\lambda^{(i)}} .
$$

Here for clarity of display, the symbol $\sum^{\star}$ is used to recall that for $r=2$ the partition $\mu_{\downarrow(2)}$ has one part 1 . Therefore because of $(5.1), \vartheta_{\mu_{\downarrow}(2)}^{\lambda}$ must be replaced by $(n-k+$ 2) $\vartheta_{\mu \backslash(2)}^{\lambda}$.

5.4. The operator $D_{1}$. For any integers $r, s \geq 1$ we define (if they exist)

$$
\mu_{\downarrow(r s)}=\mu \backslash(r, s) \cup(r+s-1), \quad \mu_{\uparrow(r s)}=\mu \backslash(r+s+1) \cup(r, s) .
$$

We have $\left|\mu_{\downarrow}(r s)\right|=\left|\mu_{\uparrow(r s)}\right|=k-1, l\left(\mu_{\downarrow(r s)}\right)=l(\mu)-1$ and $l\left(\mu_{\uparrow(r s)}\right)=l(\mu)+1$. Writing Proposition 8 for $j=1$ we readily obtain

$$
\begin{aligned}
D_{1} p_{\mu}=\sum_{r, s} r s m_{r}(\mu) & \left(m_{s}(\mu)-\delta_{r s}\right) p_{\mu_{\downarrow}(r s)}+\frac{2 N-1}{\alpha} \sum_{r} r m_{r}(\mu) p_{\mu_{\downarrow}(r)} \\
& +\frac{\beta}{\alpha} \sum_{r} r(r-1) m_{r}(\mu) p_{\mu_{\downarrow}(r)}+\frac{1}{\alpha} \sum_{r} r m_{r}(\mu) \sum_{i=1}^{r-2} p_{\mu_{\uparrow(i, r-i-1)}} .
\end{aligned}
$$


Using (5.1) and $E_{1} p_{\mu}=k p_{\mu}$, the identity of Proposition 6 writes as

$$
\begin{gathered}
\sum_{r, s} r s m_{r}(\mu)\left(m_{s}(\mu)-\delta_{r s}\right) \vartheta_{\mu_{\downarrow}(r s)}^{\lambda}+(2 N-1) \sum_{r}^{\star} r m_{r}(\mu) \vartheta_{\mu_{\downarrow}(r)}^{\lambda} \\
+\beta \sum_{r}^{\star} r(r-1) m_{r}(\mu) \vartheta_{\mu_{\downarrow}}^{\lambda}+\alpha \sum_{r}^{\star} r m_{r}(\mu) \sum_{i=1}^{r-2} \vartheta_{\mu_{\uparrow(i, r-i-1)}}^{\lambda}+(n+k+N(N-1) / \alpha) \vartheta_{\mu}^{\lambda} \\
=\alpha \sum_{i=1}^{l(\lambda)+1} c_{i}(\lambda)\left(\lambda_{i}+(N-i) / \alpha\right)\left(\lambda_{i}+(N-i+1) / \alpha\right) \vartheta_{\mu}^{\lambda^{(i)}} .
\end{gathered}
$$

As before, the symbol $\sum^{\star}$ is used to recall the occurrence of parts 1 :

(i) The partitions $\mu_{\downarrow(2)}$ and $\mu_{\uparrow(1, r-2)}(r \geq 4)$ have one part 1 . Therefore due to (5.1), $\vartheta_{\mu_{\downarrow(2)}}^{\lambda}$ must be replaced by $(n-k+2) \vartheta_{\mu \backslash(2)}^{\lambda}$ and $\vartheta_{\mu_{\uparrow(1, r-2)}}^{\lambda}$ by $(n-k+$ 2) $\vartheta_{\mu \backslash(r) \cup(r-2)}^{\lambda}$.

(ii) The partition $\mu_{\uparrow(1,1)}$ has two parts 1 . Because of $(5.2), \vartheta_{\mu_{\uparrow(1,1)}}^{\lambda}$ must be replaced by $(n-k+3)(n-k+2) \vartheta_{\mu \backslash(3)}^{\lambda}$.

Clearly if we identify the coefficients of $N^{2}$ on both sides, we recover (5.5). Then if we identify the coefficients of $N$, we recover (5.6). Finally the remaining terms give

$$
\begin{array}{r}
\sum_{r, s} r s m_{r}(\mu)\left(m_{s}(\mu)-\delta_{r s}\right) \vartheta_{\mu_{\downarrow}(r s)}^{\lambda} \\
+\beta \sum_{r}^{\star} r(r-1) m_{r}(\mu) \vartheta_{\mu_{\downarrow}(r)}^{\lambda}+\alpha \sum_{r}^{\star} r m_{r}(\mu) \sum_{i=1}^{r-2} \vartheta_{\mu_{\uparrow(i, r-i-1)}^{\lambda}}^{l(\lambda)+1} c_{i}(\lambda)\left(\lambda_{i}-(i-1) / \alpha\right)^{2} \vartheta_{\mu}^{\lambda^{(i)}} .
\end{array}
$$

All coefficients $\vartheta_{\rho}^{\lambda}$ appearing in the left-hand side correspond to partitions $|\rho|=$ $k-1$. Below this property will be crucial for our purpose.

5.5. The operator $D_{2}$. For completeness we give an analogous result for $D_{2}$, though we shall not use it.

For any integers $r, s \geq 1$ we define (if they exist)

$$
\mu_{\Downarrow(r s)}=\mu \backslash(r, s) \cup(r+s), \quad \mu_{\Uparrow(r s)}=\mu \backslash(r+s) \cup(r, s) .
$$

We have $\left|\mu_{\Downarrow(r s)}\right|=\left|\mu_{\Uparrow(r s)}\right|=k, l\left(\mu_{\Downarrow(r s)}\right)=l(\mu)-1$ and $l\left(\mu_{\Uparrow(r s)}\right)=l(\mu)+1$. Writing Proposition 8 for $j=2$, we easily obtain

$$
\begin{aligned}
\left(D_{2}^{\dagger}-\frac{\beta}{\alpha} \sum_{r} r(r-1) m_{r}(\mu)\right) p_{\mu} & = \\
& \sum_{r, s} r s m_{r}(\mu)\left(m_{s}(\mu)-\delta_{r s}\right) p_{\mu_{\Downarrow}(r s)}+\frac{1}{\alpha} \sum_{r} r m_{r}(\mu) \sum_{i=1}^{r-1} p_{\mu_{\Uparrow(i, r-i)}} .
\end{aligned}
$$


Then Proposition 7, specialized for $f=p_{\mu}$, writes as

$$
\begin{aligned}
\sum_{r, s} r s m_{r}(\mu) & \left.m_{s}(\mu)-\delta_{r s}\right) \vartheta_{\mu_{\Downarrow(r s)}}^{\lambda}+\alpha \sum_{r}^{\circ} r m_{r}(\mu) \sum_{i=1}^{r-1} \vartheta_{\mu_{\Uparrow(i, r-i)}}^{\lambda} \\
& +2 \sum_{r} r m_{r}(\mu) \vartheta_{\mu_{\uparrow(r)}}^{\lambda}+\vartheta_{\mu, 2}^{\lambda}=\left(2 \alpha d_{1}(\lambda)-\beta \sum_{r} r(r-1) m_{r}(\mu)\right) \vartheta_{\mu}^{\lambda} .
\end{aligned}
$$

Again the symbol $\sum^{\circ}$ is used to specify some particular cases :

(i) For $r \geq 3$ the partition $\mu_{\Uparrow(1, r-1)}$ has one part 1. By (5.1), $\vartheta_{\mu_{\Uparrow(1, r-1)}}^{\lambda}$ must be replaced by $(n-k+1) \vartheta_{\mu \backslash(r) \cup(r-1)}^{\lambda}$.

(ii) The partition $\mu_{\Uparrow(1,1)}$ has two parts 1 . Due to $(5.2), \vartheta_{\mu_{\Uparrow(1,1)}}^{\lambda}$ must be replaced by $(n-k+2)(n-k+1) \vartheta_{\mu \backslash(2)}^{\lambda}$.

The simplest case is given by $\mu=(2)$, i.e.

$$
2\left(\alpha d_{1}(\lambda)-\beta\right) \vartheta_{2}^{\lambda}=2 \alpha n(n-1)+4 \vartheta_{3}^{\lambda}+\vartheta_{22}^{\lambda} .
$$

As another example, for $\mu=(3,2)$ the identity writes as

$\left(2 \alpha d_{1}(\lambda)-8 \beta\right) \vartheta_{32}^{\lambda}=12 \vartheta_{5}^{\lambda}+2 \alpha(n-3)(n-4) \vartheta_{3}^{\lambda}+6 \alpha(n-4) \vartheta_{22}^{\lambda}+6 \vartheta_{42}^{\lambda}+4 \vartheta_{33}^{\lambda}+\vartheta_{322}^{\lambda}$.

\section{Rectangular shape}

We are now in a position to prove the following weak version of our conjecture for $m=1$, i.e. when $\lambda=p \times q$, the rectangular shape formed by $p$ parts equal to $q$.

Theorem 1. Let $\lambda=p \times q$ and $\mu$ a partition with $m_{1}(\mu)=0$ and $|\mu|=k \leq|\lambda|=$ $p q$. The quantity $(-1)^{k} \vartheta_{\mu}^{\lambda}$ is a polynomial in the indeterminates $(p,-q, \beta)$, with nonnegative rational coefficients.

Proof. We shall use induction on the weight $|\mu|=k$. The property is verified for $k=2$. Actually it is well known (see [11, p.384], [16, p.106] or [8, p.68]) that

$$
2 \theta_{2,1^{n-2}}^{\lambda}=2 \alpha d_{1}(\lambda)=p q(\alpha q-p-\beta) .
$$

Obviously there are only two partitions $\lambda^{(i)}$, corresponding respectively to $i=1$ and $i=p+1$. We have

$$
\begin{array}{rlrl}
\lambda^{(1)}= & (q+1, q, \ldots, q), & \lambda^{(p+1)} & =(q, \ldots, q, 1), \\
c_{1}(\lambda)=\frac{p}{p+\alpha q}, & c_{p+1}(\lambda) & =\frac{\alpha q}{p+\alpha q} .
\end{array}
$$

We consider the linear system formed by (5.5), (5.6) and (5.7). Firstly we evaluate the quantities $\vartheta_{\mu}^{\lambda^{(1)}}$ and $\vartheta_{\mu}^{\lambda^{(p+1)}}$ by solving (5.5) and (5.6). This easily yields

$$
\begin{aligned}
\vartheta_{\mu}^{\lambda^{(1)}} & =\vartheta_{\mu}^{\lambda}+\frac{\alpha}{p} \sum_{r}^{\star} r m_{r}(\mu) \vartheta_{\mu_{\downarrow}(r)}^{\lambda} \\
\vartheta_{\mu}^{\lambda^{(p+1)}} & =\vartheta_{\mu}^{\lambda}-\frac{1}{q} \sum_{r}^{\star} r m_{r}(\mu) \vartheta_{\mu_{\downarrow}(r)}^{\lambda} .
\end{aligned}
$$


Secondly we insert both values in (5.7). The sum in the right-hand side writes

$$
\alpha q^{2} c_{1}(\lambda) \vartheta_{\mu}^{\lambda^{(1)}}+p^{2} c_{p+1}(\lambda) \vartheta_{\mu}^{\lambda^{(p+1)}} / \alpha=p q \vartheta_{\mu}^{\lambda}+(\alpha q-p) \sum_{r}^{\star} r m_{r}(\mu) \vartheta_{\mu_{\downarrow}(r)}^{\lambda},
$$

so that finally (5.7) becomes

$$
\begin{aligned}
& \sum_{r, s} r s m_{r}(\mu)\left(m_{s}(\mu)-\delta_{r s}\right) \vartheta_{\mu_{\downarrow}(r s)}^{\lambda}+\beta \sum_{r}^{\star} r(r-1) m_{r}(\mu) \vartheta_{\mu_{\downarrow}(r)}^{\lambda} \\
& \quad+(p-\alpha q) \sum_{r}^{\star} r m_{r}(\mu) \vartheta_{\mu_{\downarrow}(r)}^{\lambda}+\alpha \sum_{r}^{\star} r m_{r}(\mu) \sum_{i=1}^{r-2} \vartheta_{\mu_{\uparrow(i, r-i-1)}^{\lambda}}^{\lambda}=-k \vartheta_{\mu}^{\lambda}
\end{aligned}
$$

This is an inductive formula expressing $k \vartheta_{\mu}^{\lambda}$ as an integral combination of $\vartheta_{\rho}^{\lambda}$ with $|\rho|=k-1$. Multiplying both sides by $(-1)^{k-1}$, we obtain that $(-1)^{k} \vartheta_{\mu}^{\lambda}$ is a polynomial in the indeterminates $(p,-q, \beta)$, with nonnegative rational coefficients.

Observe that the partitions with parts 1 create no difficulty since their contributions are respectively $-(-p q+k-2) \vartheta_{\mu \backslash(2)}^{\lambda}$ for $\mu_{\downarrow(2)},-(-p q+k-2) \vartheta_{\mu \backslash(r) \cup(r-2)}^{\lambda}$ for $\mu_{\uparrow(1, r-2)}$, and $(-p q+k-2)(-p q+k-3) \vartheta_{\mu \backslash(3)}^{\lambda}$ for $\mu_{\uparrow(1,1)}$.

We emphasize that the previous argument does not allow to conclude that the coefficients of $\vartheta_{\mu}^{\lambda}$ are integers. It remains to prove that the coefficients of $k \vartheta_{\mu}^{\lambda}$ are divisible by $k$. The recurrence (6.2) shows empirical evidence of this fact, but we are in lack of a proof.

Below are the first steps of our recurrence, for $k \leq 6$.

$$
\begin{aligned}
-\vartheta_{2}^{\lambda}= & p q(p-\alpha q+\beta), \\
-\vartheta_{3}^{\lambda}= & \vartheta_{2}^{\lambda}(p-\alpha q+2 \beta)+\alpha p q(p q-1), \\
-\vartheta_{4}^{\lambda}= & \vartheta_{3}^{\lambda}(p-\alpha q+3 \beta)+2 \alpha(p q-2) \vartheta_{2}^{\lambda}, \\
-\vartheta_{22}^{\lambda}= & 2 \vartheta_{3}^{\lambda}+(p-\alpha q+\beta)(p q-2) \vartheta_{2}^{\lambda}, \\
-\vartheta_{5}^{\lambda}= & \vartheta_{4}^{\lambda}(p-\alpha q+4 \beta)+2 \alpha(p q-3) \vartheta_{3}^{\lambda}+\alpha \vartheta_{22}^{\lambda}, \\
-5 \vartheta_{32}^{\lambda}= & 12 \vartheta_{4}^{\lambda}+2(p-\alpha q+\beta)(p q-3) \vartheta_{3}^{\lambda} \\
& +3(p-\alpha q+2 \beta) \vartheta_{22}^{\lambda}+3 \alpha(p q-2)(p q-3) \vartheta_{2}^{\lambda}, \\
-\vartheta_{6}^{\lambda}= & \vartheta_{5}^{\lambda}(p-\alpha q+5 \beta)+2 \alpha(p q-4) \vartheta_{4}^{\lambda}+2 \alpha \vartheta_{32}^{\lambda}, \\
-6 \vartheta_{42}^{\lambda}= & 16 \vartheta_{5}^{\lambda}+2(p-\alpha q+\beta)(p q-4) \vartheta_{4}^{\lambda} \\
& +4(p-\alpha q+3 \beta) \vartheta_{32}^{\lambda}+8 \alpha(p q-4) \vartheta_{22}^{\lambda}, \\
-\vartheta_{33}^{\lambda}= & 3 \vartheta_{5}^{\lambda}+(p-\alpha q+2 \beta) \vartheta_{32}^{\lambda}+\alpha(p q-3)(p q-4) \vartheta_{3}^{\lambda}, \\
-\vartheta_{222}^{\lambda}= & 4 \vartheta_{32}^{\lambda}+(p-\alpha q+\beta)(p q-4) \vartheta_{22}^{\lambda} .
\end{aligned}
$$

Proposition 9. Let $\Lambda$ be a partition obtained by adding or substracting one node to the rectangular shape $\lambda=p \times q$. The quantity $(-1)^{k} \vartheta_{\mu}^{\Lambda}$ is a polynomial in the indeterminates $(p,-q, \beta)$, with nonnegative rational coefficients.

Proof. From the recurrence formula (6.2), it is clear that any $\vartheta_{\mu}^{\lambda}$ is divisible by $p q$. Thus by $(6.1)$, the assertion is true for both partitions $\lambda^{(1)}=(q+1, q, \ldots, q)$ and 
$\lambda^{(p+1)}=(q, \ldots, q, 1)$. Obviously there is only one partition $\lambda_{(i)}$, corresponding to $i=p$. Then we have $\lambda_{(p)}=(q, \ldots, q, q-1)$ and $\left(\begin{array}{c}\lambda \\ \lambda_{(p)}\end{array}\right)=p q$. Therefore (5.3) writes

$$
(p q-k) \vartheta_{\mu}^{\lambda}=p q \vartheta_{\mu}^{\lambda(p)} .
$$

Hence the statement for $\lambda_{(p)}$.

\section{Another formulation}

It is worth showing why the proofs given for $\alpha=1[15,17]$ are difficult to extend when $\alpha$ is arbitrary. This will also produce a non trivial result.

The proof given in [15] starts from the following formula, proved in [13, (15.21)]:

$$
\widehat{\chi}_{\mu, 1^{n-k}}^{\lambda}=\sum_{|\rho|=k} s_{\rho}^{\dagger}(\lambda) \chi_{\mu}^{\rho} .
$$

Here $s_{\rho}^{\dagger}$ is an appropriate normalization of the shifted Schur function, i.e. the shifted Jack polynomial corresponding to $\alpha=1$. Hence $s_{\rho}^{\dagger}(\lambda)$ is, up to some normalization, the generalized binomial coefficient $\left(\begin{array}{l}\lambda \\ \rho\end{array}\right)$ for $\alpha=1$.

Due to (1.1) this can be rewritten under the form

$$
\left(\begin{array}{c}
n-k+m_{1}(\mu) \\
m_{1}(\mu)
\end{array}\right) \theta_{\mu, 1^{n-k}}^{\lambda}=\sum_{|\rho|=k}\left(\begin{array}{c}
\lambda \\
\rho
\end{array}\right) \theta_{\mu}^{\rho} .
$$

It is a remarkable fact that this property keeps true when $\alpha$ is arbitrary. Actually it is exactly (3.3) written for $r=k$.

Moreover for $\lambda=p \times q$ the binomial coefficients $\left(\begin{array}{c}\lambda \\ \rho\end{array}\right)$ are explicitly known for any $\rho$. They have been computed, in the more general context of Macdonald polynomials, in $[7$, Theorem 11, p.313]. As a limit case $[7$, p.321] we have

$$
\left(\begin{array}{c}
p \times q \\
\rho
\end{array}\right)=(-\alpha)^{|\rho|}(-q)_{\rho} J_{\rho}^{\sharp}\left(1^{p}\right)=(-1)^{|\rho|} \alpha^{2|\rho|}(-q)_{\rho} \frac{(p / \alpha)_{\rho}}{j_{\rho}},
$$

the last equality being a consequence of (2.1).

Thus for $\lambda=p \times q$ we have

$$
\left(\begin{array}{c}
p q-k+m_{1}(\mu) \\
m_{1}(\mu)
\end{array}\right) \theta_{\mu, 1^{p q-k}}^{p \times q}=(-1)^{k} \alpha^{2 k} \sum_{|\rho|=k}(p / \alpha)_{\rho}(-q)_{\rho} \frac{\theta_{\mu}^{\rho}}{j_{\rho}} .
$$

Finally Theorem 1 appears equivalent to the following result, which seems difficult to prove directly (the case $m_{1}(\mu) \neq 0$ is handled by the argument given at the end of Section 3).

Theorem 2. Let $p, q$ be two indeterminates. For any partition $\mu$ the quantity

$$
\alpha^{2|\mu|-1} z_{\mu} \sum_{|\rho|=|\mu|}(p)_{\rho}(q)_{\rho} \frac{\theta_{\mu}^{\rho}}{j_{\rho}}
$$

is a polynomial in $(p, q, \beta)$ with nonnegative rational coefficients. 
We conjecture (i) the integrality of these coefficients, (ii) the existence of some natural expression as a polynomial in $(p, q, \alpha, \beta)$. However we have no conjectured combinatorial interpretation for such an expression.

As an example, for $\mu=(3,2)$ we have

$$
\begin{gathered}
6 \alpha^{9} \sum_{|\rho|=5}(p)_{\rho}(q)_{\rho} \frac{\theta_{32}^{\rho}}{j_{\rho}}=p q\left(\left(p^{4} q+4 p^{3} q^{2}+4 p^{2} q^{3}+p q^{4}\right) \alpha^{4}+\left(4 p^{3} q+9 p^{2} q^{2}+4 p q^{3}\right) \alpha^{3} \beta\right. \\
+5\left(p^{2} q+p q^{2}\right) \alpha^{2} \beta^{2}+2 p q \alpha \beta^{3}+\left(6 p^{3}+31 p^{2} q+31 p q^{2}+6 q^{3}\right) \alpha^{3} \\
\left.+\left(30 p^{2}+79 p q+30 q^{2}\right) \alpha^{2} \beta+48(p+q) \alpha \beta^{2}+24 \beta^{3}+18(p+q) \alpha^{2}+24 \alpha \beta\right) .
\end{gathered}
$$

\section{Final remarks}

We conclude by two remarks. Firstly our results for $\lambda=p \times q$ suggest the existence of some pattern underlying the theory of Jack polynomials, with $\alpha$ and $\beta=\alpha-1$ playing similar roles. Actually the recurrence formula (6.2) gives an expression of $(-1)^{k} \vartheta_{\mu}^{\lambda}$ as a "positive" polynomial in both $\alpha$ and $\beta$.

As mentioned in the introduction, we conjecture that this property is general, i.e. that for any $\lambda=\boldsymbol{p} \times \boldsymbol{q}$ the quantity $(-1)^{k} \vartheta_{\mu}^{\lambda}$ has some natural expression as a polynomial in $(\alpha, \beta, \boldsymbol{p},-\boldsymbol{q})$ with nonnegative integer coefficients.

Secondly in this article $\beta=\alpha-1$ was of course never considered as being independent of $\alpha$. However in a subsequent paper [9], we have given an algorithm allowing to define the quantities $\vartheta_{\mu}^{\lambda}$ when $(\alpha, \beta)$ are two independent parameters. It would be interesting to investigate this more general situation. This might lead to a two-parameters $(\alpha, \beta)$-extension of Jack polynomials.

\section{References}

[1] V. Féray, Proof of Stanley's conjecture about irreducible character values of the symmetric group, Ann. Comb. (2008), to appear, ArXiv math.CO/0612090.

[2] J. Kaneko, Selberg integrals and hypergeometric functions associated with Jack polynomials, SIAM J. Math. Anal. 24 (1993), 1086-1110.

[3] F. Knop, S. Sahi, Difference equations and symmetric polynomials defined by their zeros, Int. Math. Res. Not. 10 (1996), 473-486.

[4] F. Knop, S. Sahi, A recursion and a combinatorial formula for Jack polynomials, Invent. Math. 128 (1997), 9-22.

[5] M. Lassalle, Une formule de Pieri pour les polynômes de Jack, C. R. Acad. Sci. Paris, Sér. I Math., 309 (1989), 941-944.

[6] M. Lassalle, Une formule du binôme généralisée pour les polynômes de Jack, C. R. Acad. Sci. Paris, Sér. I Math., 310 (1990), 253-256.

[7] M. Lassalle, Coefficients binomiaux généralisés et polynômes de Macdonald, J. Funct. Anal. 158 (1998), 289-324.

[8] M. Lassalle, Some combinatorial conjectures for Jack polynomials, Ann. Comb. 2 (1998), 61-83.

[9] M. Lassalle, Jack polynomials and free cumulants, ArXiv 0802.0448.

[10] M. Lassalle, available at http://igm.univ-mlv.fr/ lassalle/conj.html

[11] I. G. Macdonald, Symmetric functions and Hall polynomials, Clarendon Press, second edition, Oxford, 1995.

[12] A. Okounkov, G. Olshanski, Shifted Jack polynomials, binomial formula and applications, Math. Res. Lett. 4 (1997), 69-78.

[13] A. Okounkov, G. Olshanski, Shifted Schur functions, St. Petersburg Math. J. 9 (1998), 239-300. 
[14] A. Okounkov, (Shifted) Macdonald polynomials, q-integral representation and combinatorial formula, Compos. Math. 112 (1998), 147-182.

[15] A. Rattan, Positivity results for Stanley's character polynomials, J. Algebra 308 (2007), 26-43.

[16] R. P. Stanley, Some combinatorial properties of Jack symmetric functions, Adv. Math. 77 (1989), 76-115.

[17] R. P. Stanley, Irreducible symmetric group characters of rectangular shape, Sém. Lothar. Combin. 50 (2003), article B50d.

[18] R. P. Stanley, A conjectured combinatorial interpretation of the normalized irreducible character values of the symmetric group, ArXiv math.CO/0606467.

Centre National de la Recherche Scientifique, Institut Gaspard-Monge, Université de Marne-la-Vallée, 77454 Marne-la-Vallée Cedex, France

E-mail address: lassalle@univ-mlv.fr

$U R L:$ http://igm.univ-mlv.fr/ ${ }^{\sim a s s a l l e}$ 